\title{
A Kampo Medicine, Boi-ogi-to, Inhibits Obesity in Ovariectomized Rats
}

\author{
Jun-ichi Yamakawa', Junji Moriya ${ }^{1}$, Takashi Takahashi ${ }^{1}$, Atsushi Ishige ${ }^{2}$, \\ Yoshiharu Motoo ${ }^{3}$, Fumihiko Yoshizaki ${ }^{4}$ and Tsugiyasu Kanda ${ }^{1}$
}

${ }^{1}$ Department of General Medicine, Kanazawa Medical University, Ishikawa, ${ }^{2}$ Department of Kampo Medicine, Keio University School of Medicine, Tokyo, ${ }^{3}$ Department of Medical Oncology, Kanazawa Medical University, Ishikawa and ${ }^{4}$ Department of Pharmacognosy, Tohoku Pharmaceutical University, Miyagi, Japan

\begin{abstract}
In women facing menopause, end of menstrual activity is accompanied by lower levels of estrogen and gradual weight gain. Postmenopausal weight gain sounds an alarm for women's health and may lead to hyperlipidemia, a lipid increase and glucose intolerance. These phenomena are connected to lifestyle-related diseases such as hypertension, type II diabetes mellitus, arteriosclerosis and metabolic syndrome, making it essential to prevent weight gain in women. A Kampo medicine, Boi-ogi-to, is traditionally used to treat obese conditions, but the mechanism has not yet been investigated. In this experiment, we tested the antiobesity properties of Boi-ogi-to in ovariectomized rats by measuring changes of serum cytokine levels and adipocytokines in fat cells. After treatment with this extract for 6 weeks (20-week-old rats), we found that there was a significant weight decrease in rats treated with Boi-ogi-to as compared with that in the control group. Serum tumor necrosis factor (TNF)- $\alpha$ levels increased significantly in a dose-dependent manner. Gene expression of adipose tissue in uterus also dose dependently showed a significant increase of TNF- $\alpha$ levels, suggesting that secretion of TNF- $\alpha$ by fat cells might play a role in the ability of Boi-ogi-to to inhibit weight gain. While peroxisome proliferators-activated receptor- $\gamma$ and adiponectin levels did not show a significant difference as compared with those in the control, levels of mRNA expression showed a tendency to increase dose dependently. Resistin did not show any significant change. These results suggest that Boi-ogi-to might be useful for the prevention of obesity that occurs in women with reduction of estrogen.
\end{abstract}

Keywords: Adipocytokines-Boi-ogi-to-obesity-ovariectomized rats

\section{Introduction}

For menopausal women, end of menstrual activity is accompanied by lower levels of estrogen and gradual weight gain. This postmenopausal weight gain sounds an alarm for women's health (1) since it can lead to hyperlipidemia, a lipid increase and abnormalities in

For reprints and all correspondence: Jun-ichi Yamakawa, MD, Department of General Medicine, Kanazawa Medical University, 1-1 Daigaku, Uchinada-machi, Kahoku-gun, Ishikawa 920-0293, Japan.

Tel: + 81-76-286-2211(ext. 3843); Fax: + 81-76-286-2702;

E-mail: yamakawa@kanazawa-med.ac.jp blood sugar concentrations. These phenomena are all connected to lifestyle-related diseases such as hypertension, type II diabetes mellitus, arteriosclerosis and metabolic syndrome $(2,3)$ making it essential to prevent weight gain that occurs as the hormone levels are reduced.

Ovariectomized rats (OVR) are wellknown as a model for obesity from limited estrogen function $(4,5)$. The recent report has drawn attention to the connection of leptin secretion from the lower thalamus with obesity (6). Other reports have pointed to a connection between gonadotropins from estrogen and resistin (7). 
The decrease in estrogen levels after the removal of ovaries has been shown to have a direct influence on fat cells and subsequent obesity (8).

Plants were, and still are, widely used for a number of conditions affecting women in California. Adams and Garcia (9) discuss traditional remedies of the Chumash for dysmenorrhea, premenstrual syndrome, feminine hygiene, heavy menstruation, urinary tract infections, parturition, lactation, infant care, menopause, sexually transmitted diseases, fertility, contraception and abortions. Two Kampo medicines, Bofu-tsusho-san and Oren-gedoku-to, have revealed an ability to inhibit weight gain $(4,5)$. Another Kampo medicine, Boi-ogi-to has been traditionally used to treat obese conditions. Boi-ogi-to is a component crude herbal drug that contains Sinomenium stem, Astragali radix, Aractylodis rhizome, Gycyrrhizae radix, Ziniberis rhizome and Zizyphi fructus. Boi-ogi-to is shown to increase blood insulin levels and to fall blood glucose concentrations in streptozotocin-induced, diabetic ddY mice. These actions are promoted by $A$. radix included in Boi-ogi-to (10). This agent has been found to be especially suited to those with a rounded body type characterized by soft muscles, water retention, easily tiring and the tendency to have edema in the lower extremities and knee joints in some clinical situations.

These characteristics are often found in middle-aged women and obese children. Since the mechanism for the efficacy of this component herb has not yet been discovered, the purpose of our investigation was to confirm the efficacy of Boi-ogi-to for obesity accompanying menopause. Using a model of OVR, we analyzed the changes in various cytokines' levels in the blood and influence on the adipocytokines of lipid cells in the uterus.

\section{Materials and Methods}

\section{Experimental Animals, Diet and Living Conditions}

Ovariectomy was performed for 7-week-old SpragueDawley rats (CLEA, Japan, Inc.) (11,12). After removing the ovaries, the rats were kept for 7 weeks and were divided into four groups comprising six rats each to measure the average weight. The control group (ovx-C) was fed CE-2 (CLEA Rodent Diet CE-2) from birth until 14 weeks of age. The other three groups received a mixture of rodent chow and Boi-ogi-to. The ovx-1\% group had $1 \%$ Boi-ogi-to mixed in with the rodent chow, and the ovx- $0.5 \%$ group included $0.5 \%$ Boi-ogi-to mixed with it, and the ovx $-0.25 \%$ population did $0.25 \%$ Boi-ogi-to.

While undergoing these feeding regimens, weight and the amount of chow consumed were measured once a week, at which time the chow was also changed.
The rats were raised at room temperature $23 \pm 1{ }^{\circ} \mathrm{C}$, humidity of $55 \pm 5 \%$, circadian cycle was $12 \mathrm{~h}$ of darkness, with the light period lasting from $7 \mathrm{a} . \mathrm{m}$. to 7 p.m., and were fed water and chow ad libitum.

\section{Boi-ogi-to Extract}

The component herbal remedy Boi-ogi-to was applied in this investigation. Clinically, a 1:1 combination ratio of Shakuyaku and Kanzo is applied based on Shokanron (Shang han lun: a classic textbook for acute infectious disease of traditional Chinese medicine). It contains S. $(27.0 \%), A$. radix $(27.0 \%), A$. rhizome $(16.2 \%)$, G. radix $(8.1 \%), Z$. rhizome $(5.4 \%)$ and $Z$. fructus $(16.2 \%)$. Clinical indications for treatment with this regent have been as follows: acute to chronic nephritis, nephritic syndrome, infectious anaphylaxis, cardiac and renal edema, osteoarthritis, rheumatoid arthritis, neuralgia and hyperidosis. Since Kampo medicines are made of natural herbs, the effective components might change, making it difficult to achieve repeatability in experiments. To maintain the effective amounts of this extract in our experiment, we used freeze-dried derivatives of the Kampo extract (Tsumura Co., Tokyo, Japan). The quality data regarding a3D, high-performance liquid chromatography of the constituents are also indicated in Fig. 1.

\section{Experimental Procedures}

The rat weight and dietary consumption were measured weekly after the age of 10 weeks. Each rat was kept in its own cage and 3 months after beginning the experiment ( 26 weeks of age), the rats were fasted for $24 \mathrm{~h}$ before collecting blood samples and blood was taken from their jugular veins. Two milliliters of blood were taken from the rat's jugular vein, $1 \mathrm{ml}$ was prepared by centrifugation and divided into two parts of $500 \mu 1$ each and frozen and stored at $80^{\circ} \mathrm{C}$. Afterwards, they were anesthetized with ether and their abdomens were opened, removing the fat tissues, the liver, heart and spleen and then weighing them. Thereafter, we divided the tissues and organs into thirds, freezing two parts at $80^{\circ} \mathrm{C}$ and fixing one part in formalin.

\section{Cytokine Measurements}

Adiponectin, TNF- $\alpha$, interferon (INF) $-\gamma$, interleukin (IL)-10, IL-6, IL-4, IL-2, IL- $1 \alpha$, IL-1 $\beta$ and granulocytemacrophage colony-stimulating factor (GM-CSF) were measured with a rat cytokine assay according to the protocol of a suspension array system (Japanese Bio-Rad Co.) 


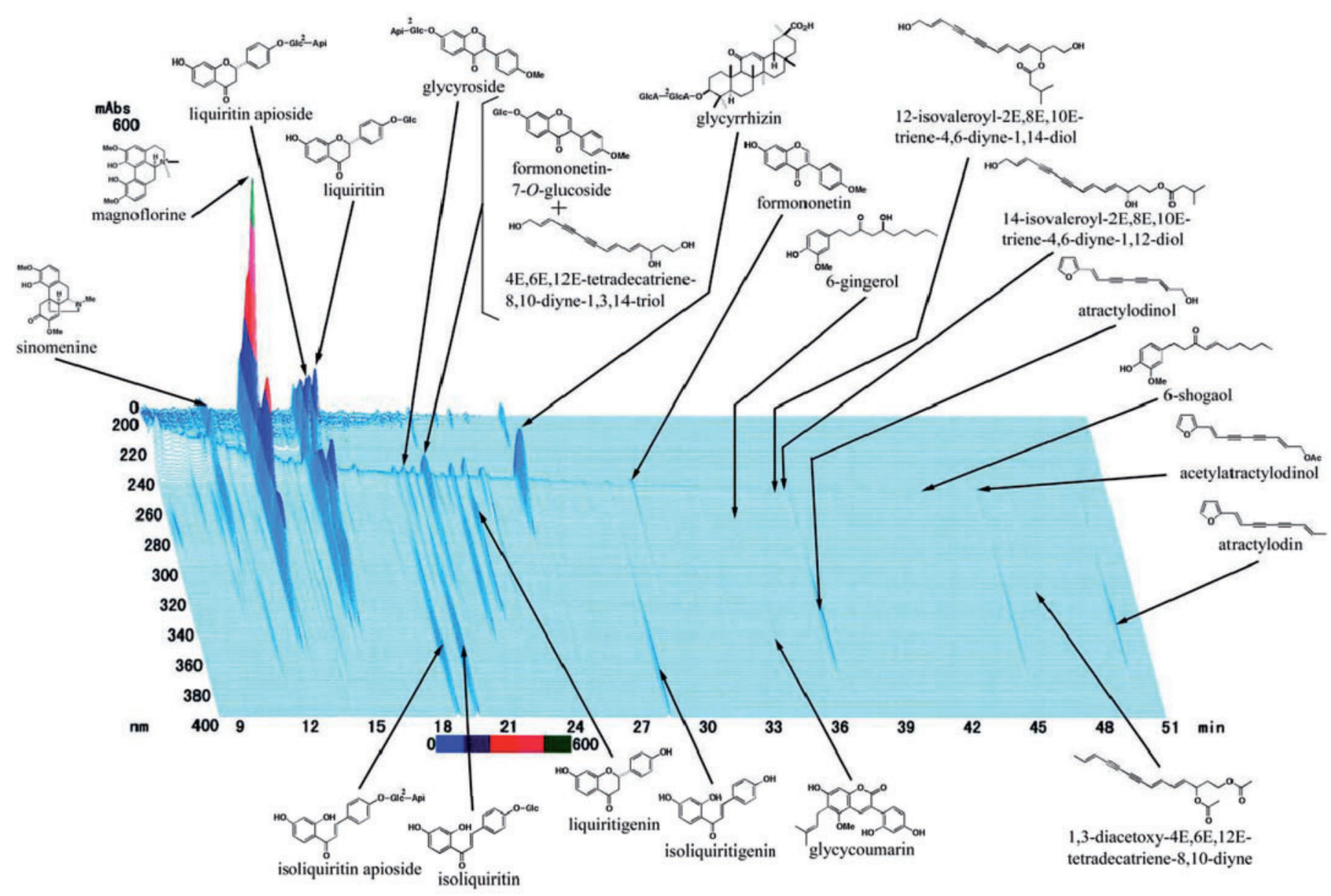

Figure 1. Pattern of 3D, high-performance liquid chromatography of the Boi-ogi-to constituents.

\section{Gene Expression Analyses}

Using RNeasy Lipid Tissue Kit (Qiagen Co.), total RNA was extracted from the fat tissues of uterus. The quality for RNA was determined by Agilent BioAnalysis. With the total RNA prepared, expression levels of mRNA of adiponectin, TNF- $\alpha$, peroxisome proliferators-activated receptor (PPAR)- $\gamma$, or resistin were determined as follows: Rattus norvegicus adiponectin receptor 1 (LOC289036) mRNA; RA015207-F [CACAGAAAC TGGCAACATCTGGA]/RA015207-R [CTGAATGAC AGTAGACGGTGTGGAA], Rattus norvegicus TNF superfamily member 2 (Tnf) mRNA; RA008379-F [AA CTCGAGTGACAAGCCCGTAG]/RA008379-R [GTAC CACCAGTTGGTTGTCTTTGA], Rattus norvegicus PPAR-gamma (Pparg) mRNA; RA008979-F [TGTCGG TTTCAGAAGTGCCTTG]/RA008979-R [TTCAGCTG GTCGATATCACTGGAG], Rattus norvegicus resistin (Retn) mRNA; RA013938-F [TCATGCCCAGAACCGA GTTG]/RA013938-R [TCATCCATGGGACACAGTGA CA]. Modified assay system for these gene expression analyses (Takara Bio Co.) using real-time polymerase chain reaction $(\mathrm{PCR})$ was used.

\section{Statistical Analyses}

Each measured value was expressed as standard deviations in the average value and significance was determined by analysis of variance (ANOVA). Using Fisher's protected LSD test, a value of $P<0.05$ was considered to be significantly different.

\section{Results}

\section{Amount of Food Consumed, Body Weight and Tissue Weights}

No significant difference in diet amount was found among the four groups (ovx-C: $23.7 \pm 0.9 \mathrm{~g} /$ day, ovx-1\%: $22.9 \pm 1.2 \mathrm{~g} /$ day, ovx $-0.5 \%$ : $23.5 \pm 1.6 \mathrm{~g} /$ day, ovx $-0.25 \%$ : $23.5 \pm 1.3 \mathrm{~g} /$ day). Changes in body weight during the observation period are shown in Fig. 2A. As compared with the control group, the ovx-1\% group showed a significant alteration to lose the weight after the 6th experimental week (the rats were 20 weeksold). The ovx$0.5 \%$ and ovx $-0.25 \%$ groups did not change significantly, suggesting that the concentrations of Boi-ogi-to might 
A

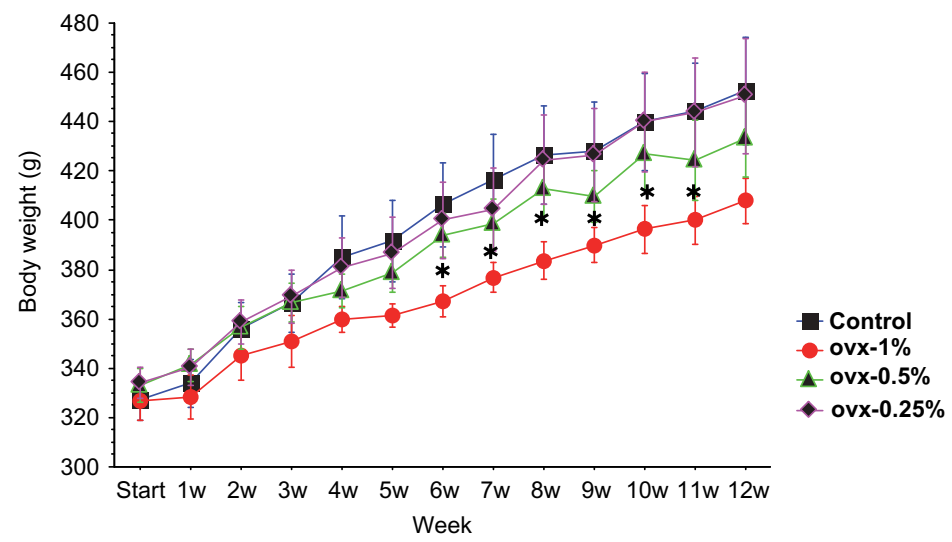

B
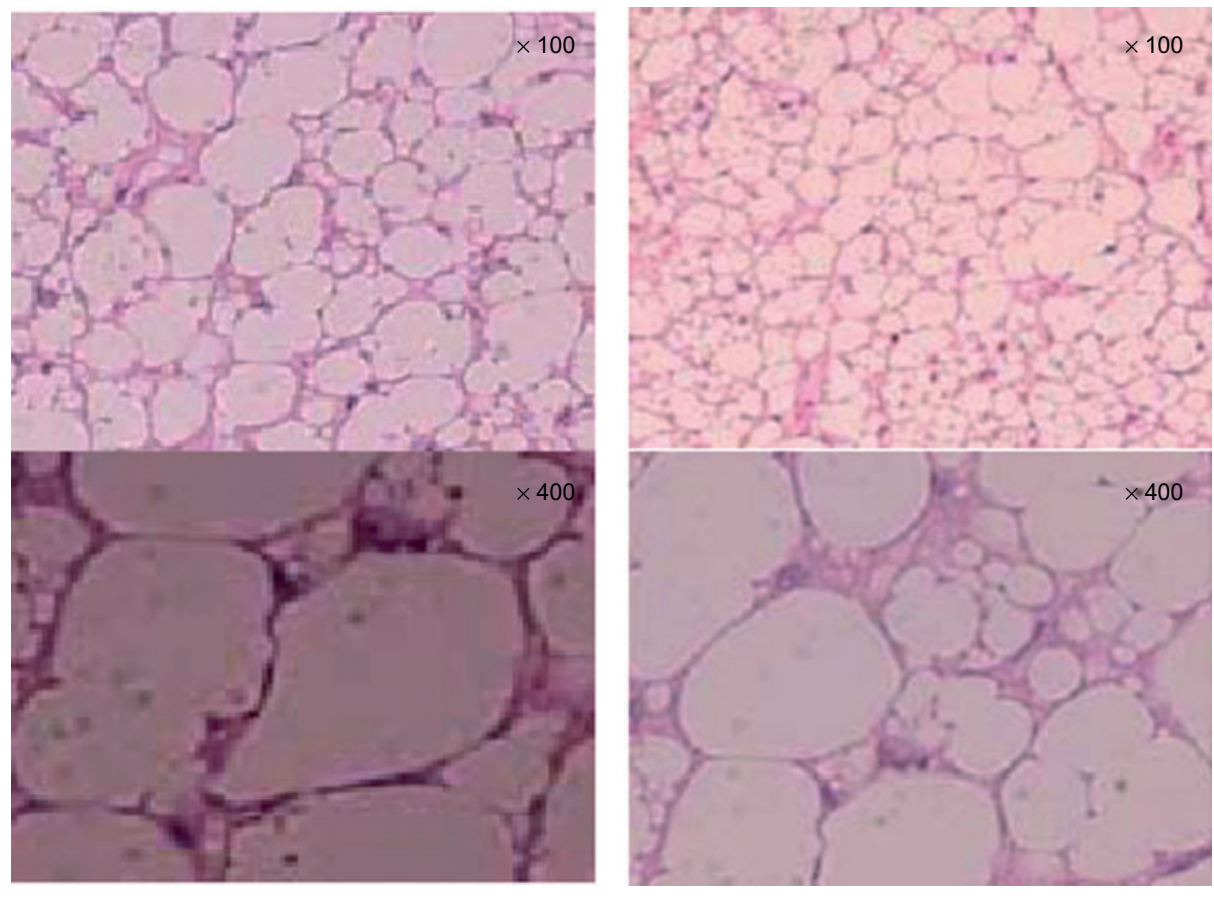

Control

ovx-1\%

Figure 2. Weekly variation of body weight among different groups from the start of treatment with Boi-ogi-to to the end of experiment (A) and findings of hematoxylin-eosin staining of adipose cells in uterus in the control and the treated group (ovx-1\%). (B) Data are expressed as means $\pm \mathrm{SD} .{ }^{*} P<0.05$ compared with that in the control. Ovx-1\% group had $1 \%$ Boi-ogi-to mixed in with the rodent chow, ovx- $0.5 \%$ group included $0.5 \%$ Boi-ogi-to mixed with it and ovx- $0.25 \%$ population included $0.25 \%$ Boi-ogi-to. The upper and lower images for histopathological examinations indicate low (100-fold) and high (400-fold) magnifications, respectively.

influence the tendency towards weight loss. After 3 months (rats were 26 weeks old), the ratios of weight of the fat tissues of uterus/body weight showed significant difference according to the concentration of Boi-ogi-to (Table 1). The ratios of liver weight/body weight and heart weight/body weight showed significant changes (Table 1). Figure 2B shows hematoxylin-eosin staining of adipose cells in the uterus in the control and the ovx-1\% group.

\section{Triglyceride, Cholesterol, Glucose or Insulin in Sera}

One and three months after the beginning of experiment (rats were 18 and 26 weeks old), blood specimens were taken after the rats had fasted for $24 \mathrm{~h}$. The results regarding serum triglyceride, cholesterol, glucose, or insulin indicated no significant differences among the different groups (Fig. 3A-D).

\section{Various Cytokine Levels in Sera}

No significant differences in serum adiponectin (Fig. 4A), INF- $\gamma$ (Table 2), GM-CSF (Table 2), IL-1 $\beta$ (Table 2), IL-2 (Table 2), IL-4 (Table 2), IL-6 (Fig. 4B) and IL-10 (Table 2) levels were found among the four groups. However, Fig. 4C shows significant elevation of serum $\mathrm{TNF}-\alpha$ concentrations in a dose-dependent manner in comparison to that in the ovx-C group. As compared 
with that in the ovx-C group, the levels of IL- $1 \alpha$ were significantly lower in both the ovx- $0.5 \%$ and ovx $-0.25 \%$ groups (Fig. 4D).

\section{Gene Expression Levels in Fat Tissues of Uterus Measured by Real-time PCR}

Using real-time PCR, we measured expression levels of the following mRNA in the fat tissues of uterus with assaying (i) adiponectin, (ii) PPAR- $\gamma$ (iii) resistin and (iv) TNF- $\alpha$. The first three parameters showed no significant changes: (i) adiponectin (Rattus norvegicus adiponectin receptor 1), (ii) PPAR- $\gamma$ ( $R$. norvegicus PPAR- $\gamma$ ) and (iii) resistin ( $R$. norvegicus resistin). The TNF- $\alpha$ ( $R$. norvegicus TNF superfamily member 2$)$, however, showed a tendency to increase dose dependently as compared with that in the control (Fig. 5A-D).

Table 1. Each ratio of various organ weight per body weight in different groups

\begin{tabular}{lllll}
\hline & Spleen & Liver & \multicolumn{1}{l}{ Heart } & Adipose tissue \\
\hline Control & $0.136 \pm 0.014$ & $2.279 \pm 0.147$ & $0.239 \pm 0.019$ & $1.981 \pm 0.129$ \\
ovx-1\% & $0.154 \pm 0.014$ & $2.179 \pm 0.15$ & $0.219 \pm 0.017$ & $1.682 \pm 0.149 *$ \\
ovx- $0.5 \%$ & $0.153 \pm 0.013$ & $2.251 \pm 0.193$ & $0.24 \pm 0.021$ & $1.589 \pm 0.166^{*}$ \\
ovx- $0.25 \%$ & $0.133 \pm 0.013$ & $2.265 \pm 0.193$ & $0.232 \pm 0.023$ & $1.607 \pm 0.213^{*}$ \\
\hline
\end{tabular}

Data are expressed as means $\pm \mathrm{SD} . * P<0.05$ compared with that in the control. Ovx-1\% group had $1 \%$ Boi-ogi-tomixed in with the rodent chow, ovx- $0.5 \%$ group included $0.5 \%$ Boi-ogi-tomixed with it and ovx$0.25 \%$ population included $0.25 \%$ Boi-ogi-to.

\section{Discussion}

No difference in the diet amount was found among the different groups, but the body weight of OVR showed a significant decrease after treatment for 6 weeks. The possible mechanism of Boi-ogi-to effects on body weight was not investigated. Regarding the doses of the herbal drug, the ovx-1\% group was administered $56.1 \mathrm{mg} / \mathrm{kg} /$ day, ovx $-0.5 \%$ was $27.1 \mathrm{mg} / \mathrm{kg} /$ day and the ovx $-0.25 \%$ group was $13.0 \mathrm{mg} / \mathrm{kg} / \mathrm{day}$, showing that weight loss might be dependent on the administrative doses. In addition, after 3 months (26-week-old rats) a significant difference could be found in the ratio of body weight/ weight of fat tissue of uterus. Based on these observations, we would further propose that Boi-ogi-to might be useful in regulating weights of fat tissues when estrogen levels are lowered.

Since there was no change in serum lipids, glucose, or insulin, insulin resistance was not found after 3 months of experiment. We did not measure any change of other lipid profile, such as free fatty acid or HDL and LDL cholesterol in all groups because we were not able to find significant difference in serum levels of triglyceride and total cholesterol.

The cytokine of IL- 1 is classified into IL- $1 \alpha$ and IL-1 $\beta$ based on difference of an isoelectric point. IL-1 is producted in every organism, and approximately $90 \%$ dose of IL- 1 is IL-1 $\beta$. IL- $1 \alpha$ is secreted in fibroblast or keratinocyte whereas IL-1 $\beta$ is produced in only phagocyte. However, there is a very limited report on
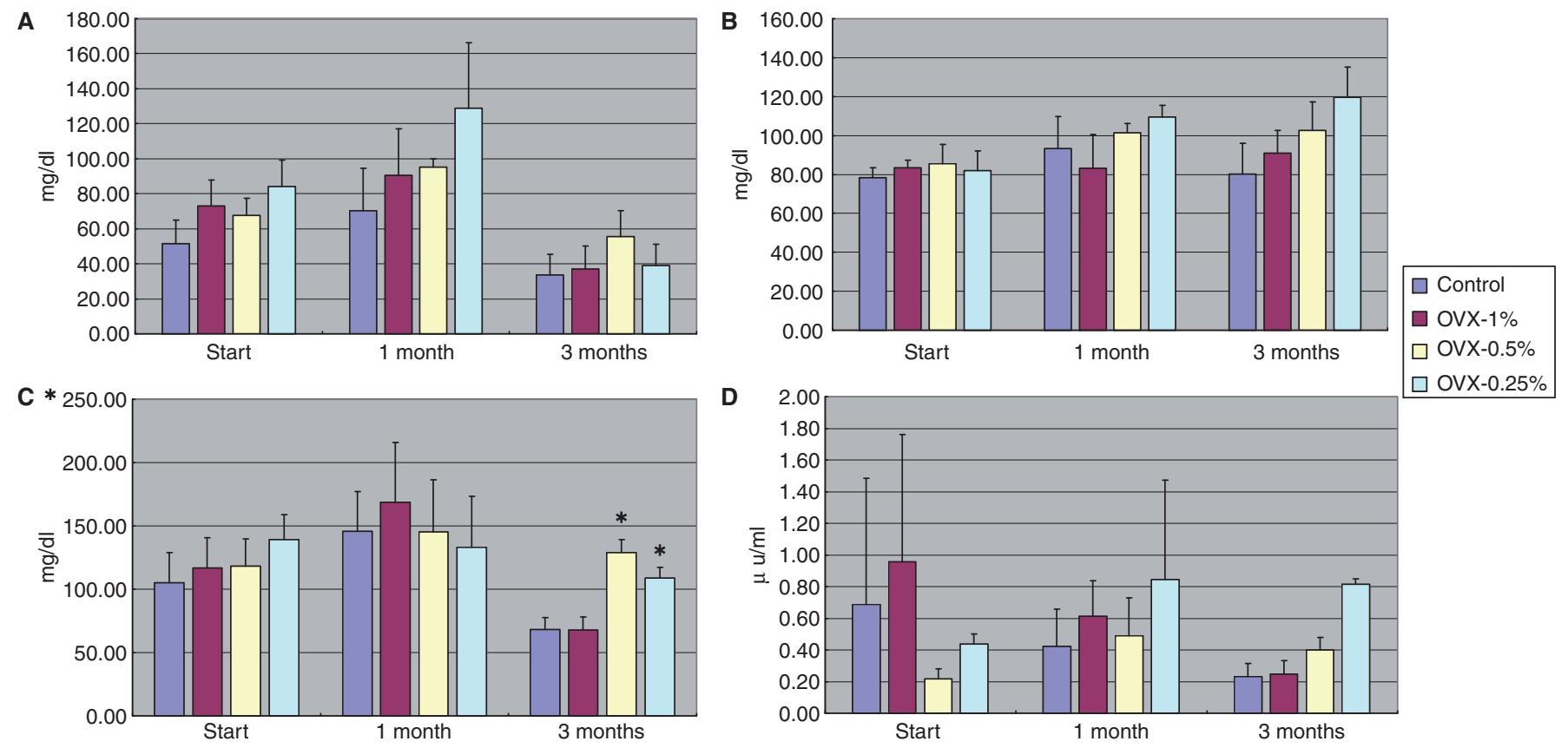

Figure 3. Serum levels of triglyceride (A), cholesterol (B), glucose (C) and insulin (D) among different groups at baseline, 1 and 3 months after treatment with Boi-ogi-to. Data are expressed as means $\pm \mathrm{SD} . * P<0.05$ compared with that in the control. Ovx-1\% group had $1 \%$ Boi-ogi-to mixed in with the rodent chow, ovx $-0.5 \%$ group included $0.5 \%$ Boi-ogi-to mixed with it and ovx- $0.25 \%$ population included $0.25 \%$ Boi-ogi-to. 

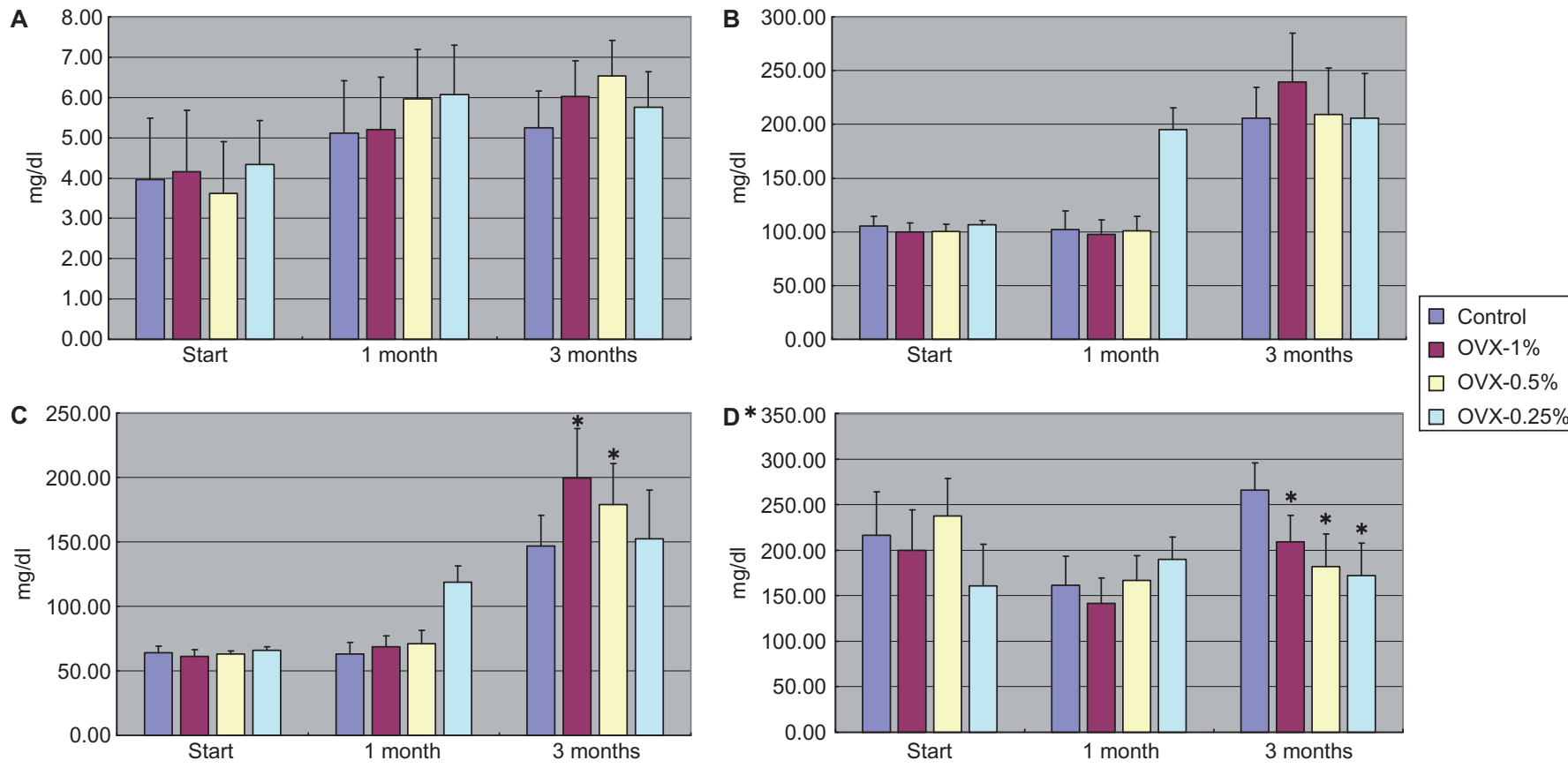

Figure 4. Serum concentrations of adiponectin (A), IL-6 (B), TNF- $\alpha(\mathbf{C})$ and IL-1 $\alpha$ (D) among different groups at baseline, 1 and 3 months after treatment with Boi-ogi-to. Data are expressed as means \pm SD. $* P<0.05$ compared with that in the control. Ovx-1\% group had $1 \%$ Boi-ogi-to mixed in with the rodent chow, ovx $-0.5 \%$ group included $0.5 \%$ Boi-ogi-to mixed with it and ovx- $0.25 \%$ population included $0.25 \%$ Boi-ogi-to.

Table 2. Serum levels of various cytokines in different groups

\begin{tabular}{|c|c|c|c|c|c|c|}
\hline & \multicolumn{3}{|l|}{ Interferon- $\gamma$} & \multicolumn{3}{|l|}{ Interleukin-1 $\beta$} \\
\hline & Baseline & 1 month & 3 months & Baseline & 1 month & 3 months \\
\hline Control & $193.1 \pm 108.2$ & $701.2 \pm 770.3$ & $476.8 \pm 300.3$ & $254.8 \pm 36.8$ & $338.7 \pm 124.9$ & $258.9 \pm 25.9$ \\
\hline ovx $-0.5 \%$ & $147.6 \pm 104.3$ & $210.1 \pm 152.1$ & $326.4 \pm 63.0$ & $429.6 \pm 28.8$ & $315.8 \pm 138.5$ & $285.4 \pm 39.8$ \\
\hline ovx- $-0.25 \%$ & $135.2 \pm 102.5$ & $483.2 \pm 150.2$ & $698.4 \pm 59.8$ & $218.3 \pm 27.1$ & $376.5 \pm 89.9$ & $375.6 \pm 46.4^{*}$ \\
\hline Control & $325 \pm 7.59$ & $286.7 \pm 13.4$ & $378.4 \pm 18.8$ & $77.0 \pm 7.97$ & $82.2 \pm 11.3$ & $208.7 \pm 31.3$ \\
\hline ovx- $1 \%$ & $330.8 \pm 7.63$ & $272.5 \pm 15.0$ & $408.7 \pm 31.6$ & $76.1 \pm 9.07$ & $95.8 \pm 11.4$ & $244.1 \pm 47.2$ \\
\hline ovx- $-0.5 \%$ & $345.8 \pm 6.29$ & $311.6 \pm 32.5$ & $425.7 \pm 28.4^{*}$ & $86.8 \pm 10.73$ & $85.7 \pm 14.9$ & $219.8 \pm 47.3$ \\
\hline \multirow[t]{2}{*}{ ovx- $0.25 \%$} & $304.7 \pm 8.15$ & $367.4 \pm 34.5^{*}$ & $417.0 \pm 32.4$ & $91.1 \pm 9.74$ & $199.4 \pm 40.13$ & $228.8 \pm 49.1$ \\
\hline & \multicolumn{3}{|l|}{ Interleukin-10 } & \multicolumn{3}{|l|}{ GM-CSF } \\
\hline ovx- $0.25 \%$ & $41.8 \pm 2.60$ & $120.1 \pm 16.2$ & $121.1 \pm 30.0$ & $72.6 \pm 6.44$ & $157.3 \pm 20.5$ & $179.4 \pm 38.8$ \\
\hline
\end{tabular}

Data are expressed as means \pm SD. $* P<0.05$ compared with that in the control. The ovx- $1 \%$ group had $1 \%$ Boi-ogi-to mixed in with the rodent chow, the ovx- $0.5 \%$ group included $0.5 \%$ Boi-ogi-to mixed with it and the ovx- $0.25 \%$ population included $0.25 \%$ Boi-ogi-to. GM-CSF, granulocyte-macrophage colony-stimulating factor.

serum levels of IL-1 $\alpha$ in the environment of obesity where Boi-ogi-to was administered. In understanding the meaning of our data regarding the IL-1 $\alpha$ levels, further investigations about the mechanisms of this cytokine production are necessary in the future.
Of the serum cytokines' concentrations, TNF- $\alpha$ levels showed a dose-dependent increase compared with the control. The IL- $1 \alpha$ levels in the ovx $-0.5 \%$ and ovx $-0.25 \%$ groups, however, showed a significant decrease as compared with the control. TNF- $\alpha$ is mainly produced 

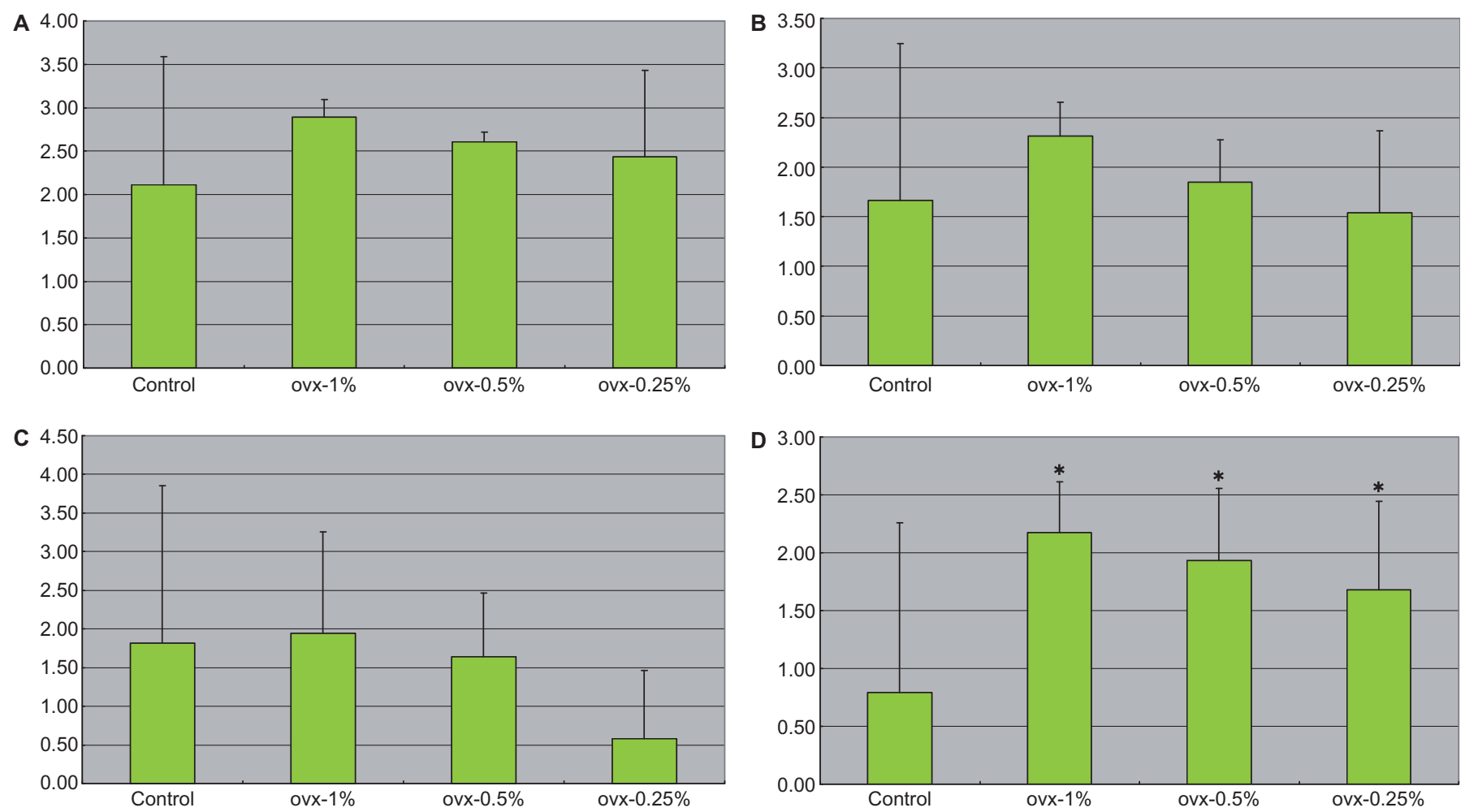

Figure 5. Gene expression levels of adiponectin (A), PPAR- $\gamma(\mathbf{B})$, resistin (C) and TNF- $\alpha$ (D) in fat tissues of uterus measured by real-time polymerase chain reaction among different groups at 3 months after treatment with Boi-ogi-to. Data are expressed as means $\pm \mathrm{SD}$. ${ }^{*} P<0.05$ compared with that in the control. Ovx- $1 \%$ group had $1 \%$ Boi-ogi-to mixed in with the rodent chow, ovx- $0.5 \%$ group included $0.5 \%$ Boi-ogi-to mixed with it and ovx- $0.25 \%$ population included $0.25 \%$ Boi-ogi-to.

by activating macrophages, and it is in part produced by the lining cells of blood vessels, adipose cells, microglia and astrocytes. With obesity, in particular, fat tissues themselves can take a central role by secreting cytokines (adipocytokines) (13) with a reaction sensitive to the metabolism. When lipid cells storing triglycerides are enlarged, the secretion of TNF- $\alpha$ is increased in the blood (14). It has been reported that TNF- $\alpha$ production in fat cells increases the concentration of TNF- $\alpha$ in the adipose tissues as well as in the blood, and can lead to improvement of insulin resistance (15). Furthermore, some reports have shown that TNF- $\alpha$ secreted into the blood by lipid cells suppresses the activation of tyrosine kinase through insulin receptors and deteriorates glucose transport function leading to insulin resistance $(16,17)$, making it important to confirm the site of TNF- $\alpha$ production.

In order to clarify the site of TNF- $\alpha$ secretion, we used an RNA preparation kit for lipids to extract the total RNA from fatty tissues in the uterus. Our results showed a significant dose-dependent elevation of TNF- $\alpha$ expression in the treated groups compared with the control, leading us to surmise that the ability of Boi-ogi-to to inhibit weight gain was connected with the secretion of TNF- $\alpha$ by lipid cells. Although several investigations $(16,17)$ reported that the secretion of TNF- $\alpha$ into the blood increases when lipid cells storing triglycerides are enlarged, the adipose cells were not enlarged and there were no changes in serum lipids, glucose, or insulin levels in our investigation, leading us to think that we would not confirm the advance of insulin resistance. From a molecular biological standpoint, the rise in TNF- $\alpha$ may be associated with cell growth since TNF- $\alpha$ is an inhibitor of cell mitosis (18). It is possible that by inhibiting the enlargement of lipid cells Boi-ogi-to facilitates the secretion of TNF- $\alpha$, an inhibitor of cell mitosis, and thereby prevents an increase in fatty tissue. Boi-ogi-to may stimulate lipid cells to secrete TNF- $\alpha$ that then inhibits cell mitosis. The second possibility is that TNF- $\alpha$ induces the activation of cyclooxygenase (COX)-2 that then produces prostaglandin (PG) (19). COX-2 is produced transiently inside the nucleus, and when inductive enzymes in the nuclear membrane stimulate the expression of inflammation-related cells, the synthesis of COX-2 is further enhanced. Important products such as $\mathrm{PGE}_{2}$ increase the intracellular levels of cAMP and cAMP can induce COX-2 through positive feedback. It is possible that the increase in cAMP levels activates the consumption of energy in lipid cells and inhibits their enlargement. The third possibility is that Boi-ogi-to works through the immune system to stimulate lipid cells to secrete TNF- $\alpha$ and that the measurement occurred 
when the secretion was excited. In addition to lipid cells, inflammatory cytokines are also produced by activated macrophages and activated linings of blood vessels, with a close connection to IL-1, IL-6, INF- $\gamma$ and IL-8. As for anti-inflammatory cytokines, IL-4, IL-10 and TNF- $\alpha$ are also concerned with the activated macrophages. In this experiment, we were unable to deny their connection as a factor, but there is a possibility that TNF- $\alpha$ is not directly connected with the inhibition of fat.

There is a limitation in this experiment. We were unable to clarify the mechanism of Boi-ogi-to action. Compared with those in the control, PPAR- $\gamma$ and adiponectin levels in sera did not show significant difference, but the expression of mRNA showed a dose-dependant tendency to increase. Resistin levels, however, showed no significant change.

Efficacy of Bofu-tsusho-san as well as Boi-ogi-to to inhibit weight gain has been indicated. Its weight loss efficacy is derived from activating brown fat tissues in organs generating heat. The metabolism of whole body is activated by enhancing the activation of brown fat tissues in organs generating heat, and then weight loss is developed. When Yoshida et al. (20) investigated effects of this drug in an obese mouse model, they showed that one of the ingredients Ephedra herb of Bofu-tsusho-san contains ephedrine, which stimulates the sympathetic nerves. It also contains phosphodiesterase inhibitors, such as Licorice, Shizonepetae spica and Forsythia, which degrade fat and activate heat generation in brown fat tissues, leading to weight loss (1).

Oren-gedoku-to acts on 3T3-L1, precursors of fat cells to control the specialization of fat cells. Its efficacy is mainly due to the action of berberine from Coptis rhizome and Phellodendri cortex that controls the expression of PPAR- $\gamma$ (2). Berberines have also exhibited antidiabetic properties in an insulin-resistant animal model. Furthermore, berberine is related to the genes of fat formation and becomes involved in the energy consumption of fat tissues by downregulating or upregulating expression (2). The components of each of these Kampo medicines differ from those of Boi-ogi-to. Among the components not found in Boi-ogi-to, Bofu-tsushosan contains Ephedra herb, Glycyrrhizae radix, S. spica, Forsythiae frutcus and Oren-gedoku-to includes $C$. rhizome and $P$. cortex. We could not find a significant difference in the expression of PPAR- $\gamma$ mRNA among the different groups. However, we surmised by the dose-dependent elevation in the mRNA expression that Boi-ogi-to might have the same mechanism as Orengedoku-to, although a new mechanism would explain its antiobesity function.

In discussion, we drew a conclusion that TNF- $\alpha$ partially involved in inhibiting weight gain attributed to postmenopause by Boi-ogi-to. However, data are weak to draw such conclusion. More animal experiments and clinical trials are needed for drawing such conclusion.
Medical approach is now being sought to prevent the disease onset. Concerning the increasing numbers of subjects with metabolic syndrome, Boi-ogi-to might become a strong candidate for use as a preventive regent. It would be important to further clarify the pharmacological functions of Kampo medicines (21-24) as well as Boi-ogi-to. In conclusion, Boi-ogi-to might be useful to prevent the development of obesity together with the reduction of estrogen levels.

\section{Acknowledgements}

This study was supported in part by a research grant from Grant-in-Aid for Scientific Research (C), the Ministry of Education, Science and Culture of Japan (No. 17590605, to J.Y., in 2005-06), the research fund of Japan Kampo Medicine Manufacturers Association (to J.M., in 2006) and the research fund of Institute of Kampo Medicine (Japan) (to T.T., in 2006). We would also like to thank Patty Christiena Willis for her help in editing this article.

\section{References}

1. Schneider JG, Tompkins C, Blumenthal RS, Mora S. The metabolic syndrome in women. Cardiol Rev 2006;14:286-91.

2. Kopelman PG. Obesity as a medical problem. Nature 2000;404:635-43.

3. Spiegelman BM, Flier JS. Obesity and the regulation of energy balance. Cell 2001;104:531-43.

4. Hioki C, Yoshimoto K, Yoshida T. Efficacy of Bofu-tsusho-san, an oriental herbal medicine, in obese Japanese women with impaired glucose tolerance. Clin Exp Pharmacol Physiol 2004;31:614-9.

5. Lee YS, Kim WS, Kim KH, Yoon MJ, Cho HJ, Shen Y, et al. Berberine, a natural plant product, activates AMP-activated protein kinase with beneficial metabolic effects in diabetic and insulinresistant states. Diabetes 2006;55:2256-64.

6. Meli R, Pacilio M, Raso GM, Esposito E, Coppola A, Nasti A, et al. Estrogen and raloxifene modulate leptin and its receptor in hypothalamus and adipose tissue from ovariectomized rats. Endocrinology 2004;145:3115-21.

7. Nogueiras R, Gualillo O, Caminos JE, Casanueva FF, Dieguez C. Regulation of resistin by gonadal, thyroid hormone, and nutritional status. Obes Res 2003;11:408-14.

8. Nishikawa Y, Ikegami H, Sakata M, Mizutani T, Morishige K, Kurachi $H$, et al. Ovariectomy increases the level of estrogen receptor mRNA and estrogen receptor binding sites in female rat adipose tissue. J Endocrinol Invest 1993;16:579-83.

9. Adams JD Jr, Garcia C. Women's health among the Chumash. Evid Based Complement Alternat Med 2006;3:125-31.

10. Tsutsumi T, Kobayashi S, Liu YY, Kontani H. Anti-hyperglycemic effect of fangchinoline isolated from Stephania tetrandra Radix in streptozotocin-diabetic mice. Biol Pharm Bull 2003;26:313-7.

11. $\mathrm{Ku} \mathrm{SK}$, Lee HS, Lee JH. Changes of gastrointestinal argyrophil endocrine cells in the osteoporotic SD rats induced by ovariectomy. J Vet Sci 2004;5:183-8.

12. Liu KJ, Wang WJ, Li DJ, Jin HF, Zhou WJ. Effect of Gengnianchun Recipe on bone mineral density, bone biomechanical parameters and serum lipid level in ovariectomized rats. Chin J Integr Med 2006;12:132-6.

13. Matsuzawa Y. The metabolic syndrome and adipocytokines. FEBS Lett 2006;580:2917-21.

14. Rondinone CM. Adipocyte-derived hormones, cytokines, and mediators. Endocrine 2006;29:81-90.

15. Langin D, Arner P. Importance of TNFalpha and neutral lipases in human adipose tissue lipolysis. Trends Endocrinol Metab 2006;17:314-20. 
16. Ronti T, Lupattelli G, Mannarino E. The endocrine function of adipose tissue: an update. Clin Endocrinol 2006;64:355-65.

17. Borst SE. The role of TNF-alpha in insulin resistance. Endocrine 2004;23:177-82.

18. Voss KA, Riley R, Dunn C, Christopher CJ. The role of tumor necrosis factor alpha and the peroxisome proliferator-activated receptor alpha in modulating the effects of fumonisin in mouse liver. Toxicology 2006;222:165-74.

19. Lee IY, Bae YD, Jeoung DI, Kang D, Park CH, Kim SH, et al. Prostacyclin production is not controlled by prostacyclin synthase but by cyclooxygenase- 2 in a human follicular dendritic cell line, HK. Mol Immunol 2007;44:3168-72.

20. Yoshida T, Sakane N, Wakabayashi Y, Umekawa T, Kondo M. Thermogenic, anti-obesity effects of Bofu-tsusho-san in MSG-obese mice. Int J Obes Relat Metab Disord 1995;19:717-22.
21. Wang XQ, Takahashi T, Zhu SJ, Moriya J, Saegusa S, Yamakawa J, et al. Effect of Hochu-ekki-to (TJ-41), a Japanese herbal medicine, on daily activity in a murine model of chronic fatigue syndrome. Evid Based Complement Alternat Med 2004:1:203-6.

22. Terasawa K. Evidence-based reconstruction of Kampo medicine: part I-is Kampo CAM? Evid Based Complement Alternat Med 2004;1:11-6.

23. Terasawa K. Evidence-based reconstruction of Kampo medicine: part II-the concept of Sho. Evid Based Complement Alternat Med 2004;2:119-23.

24. Terasawa K. Evidence-based reconstruction of Kampo medicine: part-III-how should Kampo be evaluated? Evid Based Complement Alternat Med 2004;3:219-22.

Received March 19, 2007; accepted August 16, 2007 


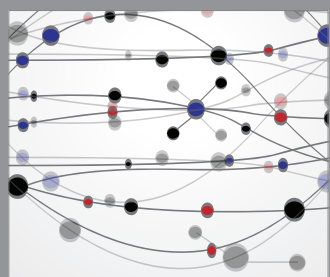

The Scientific World Journal
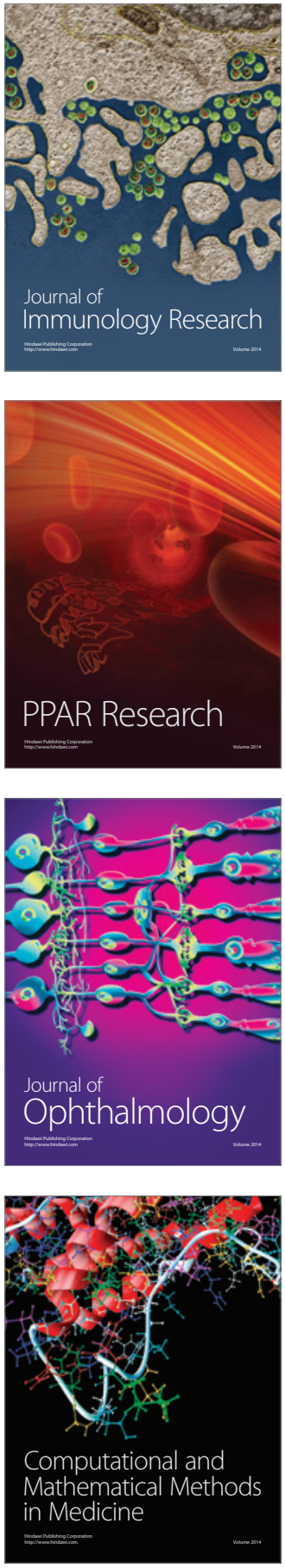

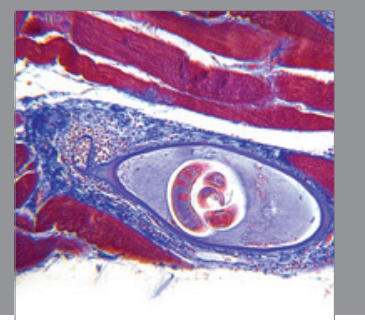

Gastroenterology

Research and Practice
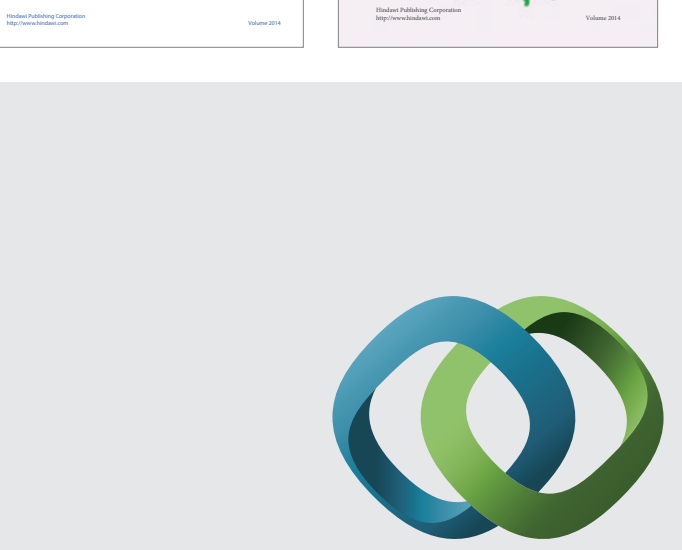

\section{Hindawi}

Submit your manuscripts at

http://www.hindawi.com
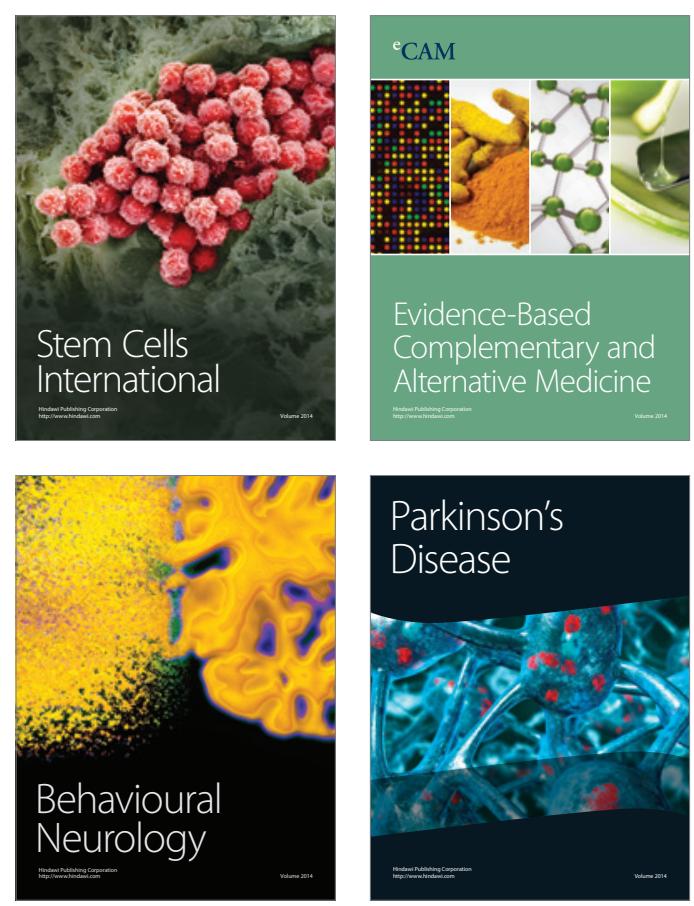

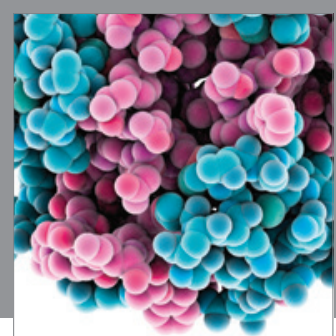

Journal of
Diabetes Research

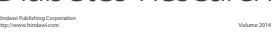

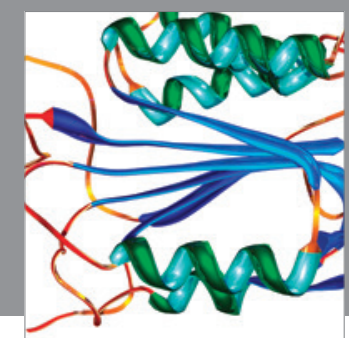

Disease Markers
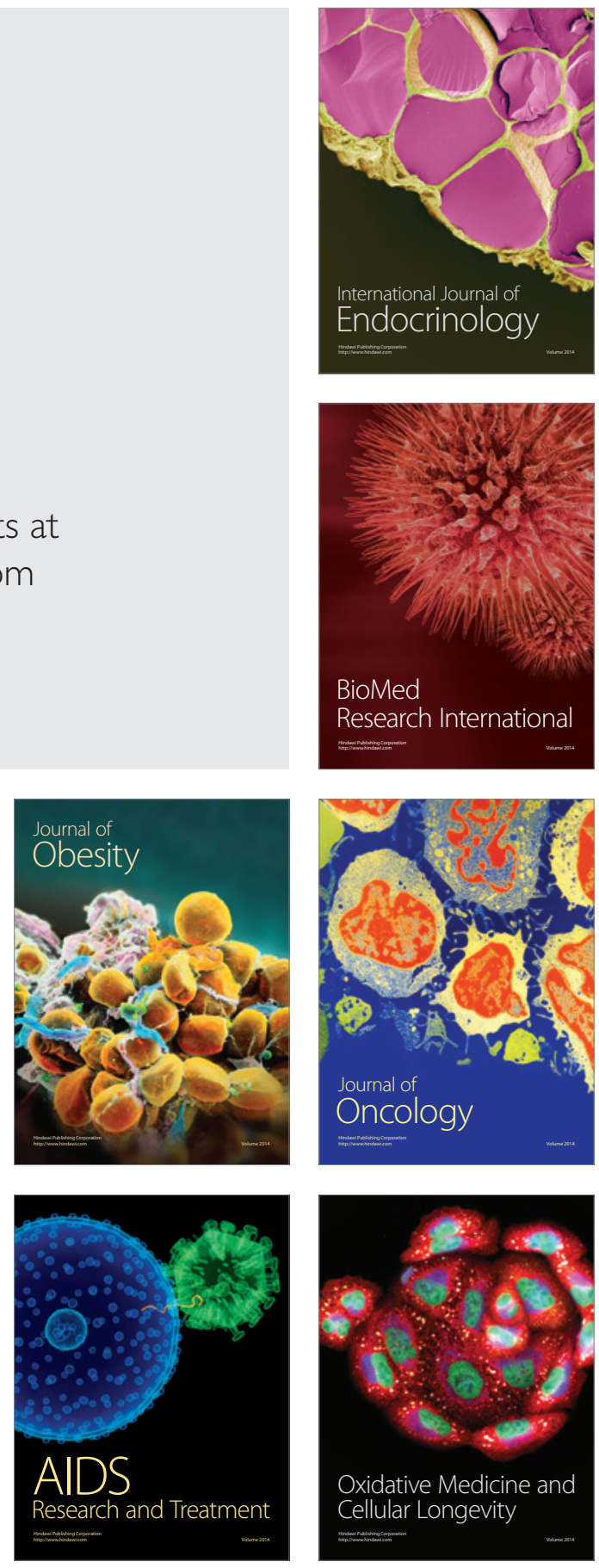